\title{
Direction Finding Using an Antenna with Direction Dependent Impulse Response
}

\author{
Heinrich Foltz \\ Electrical Engineering \\ University of Texas - Rio Grande Valley (UTRGV) \\ Edinburg, TX, USA \\ heinrich.foltz@utrgv.edu
}

\author{
Obadiah Kegege \\ Space Communications and Navigation \\ NASA Goddard Space Flight Center \\ Greenbelt, MD, USA \\ obadiah.kegege@nasa.gov
}

\begin{abstract}
Wideband antennas may be designed to have an impulse response that is direction dependent, not only in amplitude but also in waveform shape. This property can be used to perform direction finding using a single fixed antenna, without the need for an array or antenna rotation. In this paper direction finding is demonstrated using a simple candelabra-shaped monopole operating in the 1-3 GHz range. The method requires a known transmitted pulse shape and high signal-to-noise ratio, and is not as accurate or robust as conventional methods. However, it can add direction finding capability to a wideband communication system without the addition of any hardware.
\end{abstract}

\section{INTRODUCTION}

Conventional radio direction finding is performed using an array of antennas, or a single antenna that is physically rotated. Either method requires hardware in addition to that normally needed for a basic wireless communication system. The availability of wideband and ultrawideband (UWB) antennas and software-defined radios (SDR) opens up the possibility of alternative methods that can operate from a single port, nonrotating antenna attached to a single receiver front-end.

Wideband antennas can be characterized in the time domain by a normalized impulse response [[1]]. The output voltage from a receive antenna can be related to the signal input to a transmit antenna via

$$
\begin{aligned}
V_{r}(t)= & \frac{1}{2 \pi c_{0} R} h_{T}\left(\vec{a}_{r}, t\right) \otimes h_{R}\left(-\vec{a}_{r}, t\right) \\
& \otimes \frac{d V_{s}(t)}{d t} \otimes \delta\left(t-t_{d}\right)
\end{aligned}
$$

where $V_{r}$ is the received voltage, $V_{s}$ is the transmit voltage, $R$ is the distance, $h_{T}$ is the normalized input response of the transmit antenna, $h_{R}$ is the normalized input response of the receive antenna, $t_{d}$ is the total time delay, and $\vec{a}_{r}$ is a unit vector pointing from the transmit antenna to the receive antenna. Equation (1) is in a simplified form that assumes aligned polarization, a single propagation path, and equal normalizing impedances at the receive and transmit ports. As can be seen in (1), the impulse response and the waveform shape for received wideband pulses will depend on the direction of arrival $-\vec{a}_{r}$ if the other parameters are held constant, as shown in Fig. 1 Depending on the antenna design the changes in the waveform shape may be minimal (direction independent distortion [[2]]), or substantial. If the changes are substantial one can refer to antenna as having a direction dependent impulse response (DDIR), and at least in principle this property can be exploited to determine the arrival angle of incoming signals.

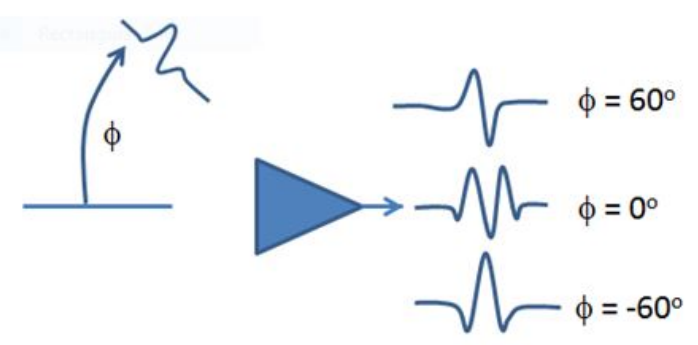

Fig. 1. Direction dependent impulse response (DDIR).

\section{ANTENNA DESIGN For DDIR}

The antenna impulse response $h_{R}$ described earlier and the transfer function $H_{R}$ are related by the usual Fourier transform relationship. One can show [[2]] that an antenna with $H_{R}$ that is a non-separable function of angle and frequency would have an impulse response that is also non-separable in angle and time, and thus has a shape that depends on angle.

In practice, one needs a reasonable level signal to analyze the shape, so the antenna must have useable gain over all angles of interest. However, if the transfer function is uniform in either angle or frequency (over the channel bandwidth), then $H_{R}$ is effectively separable and there is no DDIR effect. An initial approach to resolving this conflict is to simultaneously satisfy the following pattern characteristics: (a) an approximately omnidirectional amplitude pattern averaged over the channel bandwidth, and (b) a strongly varying phase pattern at different frequencies within the channel bandwidth.

A simple proof-of-concept prototype for the receive antenna is shown in Fig. 2. The asymmetric candelabra monopole was chosen for the first attempt due to ease of understanding and simulation. It requires a wide bandwidth (approximately 1$3 \mathrm{GHz}$ ) and needs further optimization; however, the time domain plots of output voltage in Fig. 3 definitely show 
that DDIR has been achieved. The principle of operation is that the three vertical conductors are tuned for the lower, middle, and upper portions of the band. Due to the relatively wide spacing all conductors are in play for all directions; but depending on the angle of arrival the relative group delay experienced by each of the three subbands varies significantly. This translates to ringing in the time domain, with different frequency components shifted with respect to each other in time as the angle changes.

The antenna has a single feed point in the center, with a short $(4 \mathrm{~mm})$ vertical section feeding three horizontal sections, at 120 degree spacing, each $8 \mathrm{~mm}$ wide flat strips, $4 \mathrm{~mm}$ above the ground plane, and $41 \mathrm{~mm}$ long. At the end of each horizontal section (41 mm radius from the center) is a vertical element, an $8 \mathrm{~mm}$ wide flat strip. The heights of the vertical elements (above the ground plane) are $27 \mathrm{~mm}, 41 \mathrm{~mm}$, and $63 \mathrm{~mm}$. The quarter-wave resonances for the elements independently would be at $2.77 \mathrm{GHz}, 1.83 \mathrm{GHz}$, and 1.19 $\mathrm{GHz}$, respectively.

For the experimental data shown the transmit signal was a step function fed to a 1-13 GHz dual ridged horn. Due to the rise time of the step function the majority of the spectral energy was below $3 \mathrm{GHz}$. The receiver was a broadband (DC-5 GHz) amplifier feeding a $20 \mathrm{GHz}, 50 \mathrm{GSa} / \mathrm{s}$ sampling oscilloscope. The signal-to-noise ratio for the measurement was approximately $20-25 \mathrm{~dB}$.

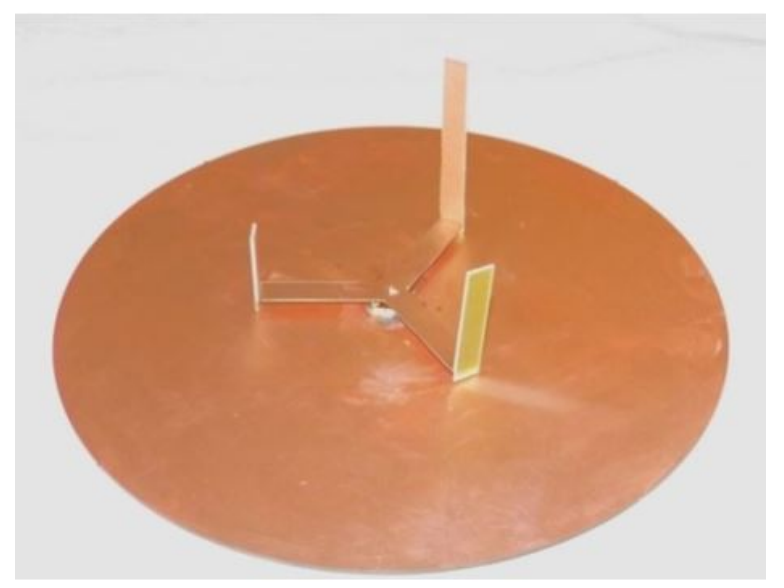

Fig. 2. Candelabra Monopole.

\section{Direction Finding Algorithm And Results}

When the electromagnetic pulse incident on the antenna has a consistent time-domain shaped, a simple algorithm is to compare the voltage output to a library of previously measured shapes at known angles. correlation with each member of a large library is computationally expensive. A related but less expensive approach is to correlate with only a few waveforms, for example, those measured previously at three known angles spaced equally in azimuth. The resulting triplet $(\alpha, \beta, \gamma)$ of real numbers forms a signature that can then be rapidly compared to a library using the magnitude of the vector difference. For

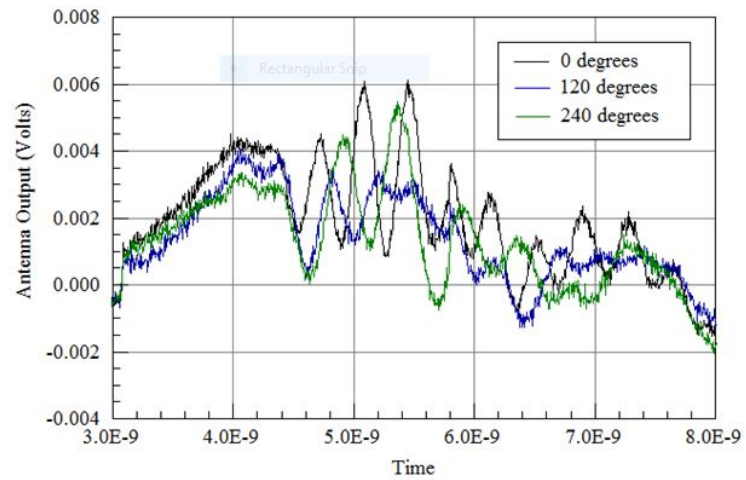

Fig. 3. Time domain output of antenna for different arrival angles.

this experiment we limited direction finding to the azimuth plane, and correlated to previously measured waveforms at $\phi=0,120$, and 240 degrees, as shown in Figure 4. The three correlation coefficients form a signature which can be used to estimate the angle of arrival. For this experiment the transmitter and receiver were triggered together and a fixed correlation was used, but in a practical situation a rolling correlation or a synchronization recovery scheme would be required.

Figure 5 shows measured values for the three correlation coefficients as a function of angle of arrival. Figure 6 shows typical accuracy data for subsequent. measurements.

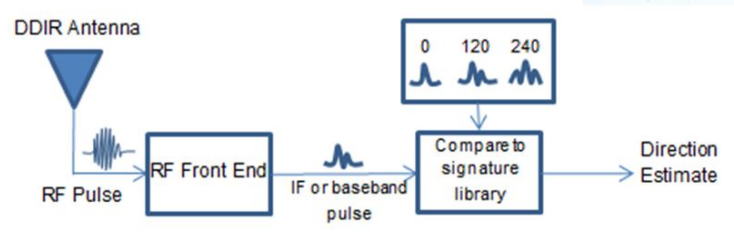

Fig. 4. Direction finding system.

\section{Discussion}

The simple algorithm used requires a known incident waveform and a relative good signal-to-noise ratio (SNR), and produces moderate levels of accuracy. Many communication systems use different waveforms to form a symbol set, in which case a larger library would be required. Since a larger number of distinct signatures would be need to resolved, an even higher higher SNR (or averaging over a larger number of pulses) would be required.

However, despite these limitations, this form of direction finding may be useful in enabling limited direction finding in systems normally intended for other purposes, especially when a broadband software defined radio is available. Since the DDIR antenna has a flat amplitude response, normal communications could use the same antenna without degradation if orthogonal frequency division multiplexing (OFDM) or similar modulation method is used. The radio could be switched to 


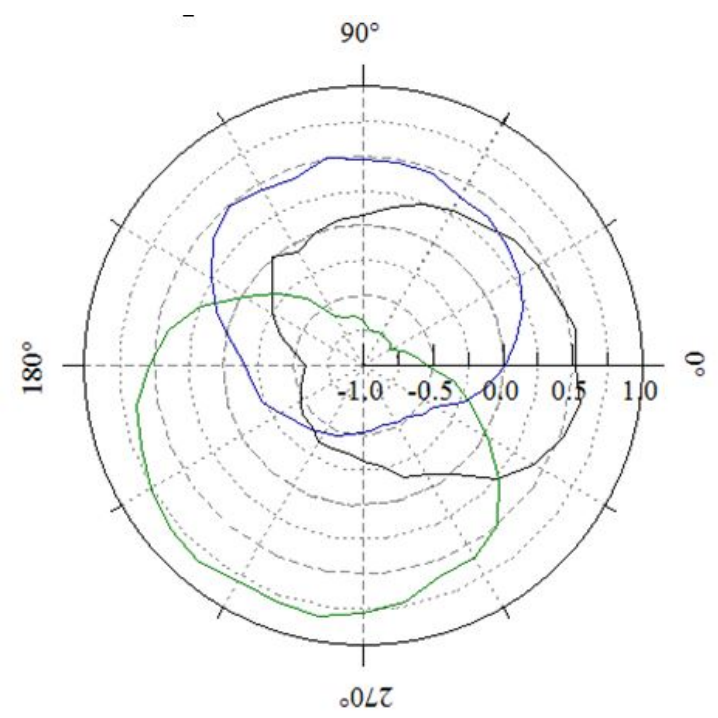

Fig. 5. Correlation of voltage waveform with known waveforms at $\phi=0$, 120,240 ; as a function of angle.

\begin{tabular}{|c|c|c|}
\hline $\begin{array}{c}\text { Actual Angle } \\
+/-3^{\circ}\end{array}$ & $\begin{array}{c}\text { DDIR } \\
\text { Estimate }\end{array}$ & Error \\
\hline $0^{\circ}$ & $353.7^{\circ}$ & $6.3^{\circ}$ \\
\hline $120^{\circ}$ & $130.3^{\circ}$ & $10.3^{\circ}$ \\
\hline $240^{\circ}$ & $255.6^{\circ}$ & $15.6^{\circ}$ \\
\hline
\end{tabular}

Fig. 6. Accuracy of direction finding.

broadband pulses when direction finding capability is desired. Typical applications would be formation flying and orientation recovery for small satellites or unmanned vehicles.

\section{ACKNOWLEDGEMENT}

The equipment used in this work was supported in part by NSF MRI Grant 0421352 and Army Research Office Grant W911NF-08-1-0192.

\section{REFERENCES}

[1] B. Scheers, M. Acheroy, A. Vander Vorst, "Time domain simulation and characterisation of TEM horns using a normalized impulse response," IEE Proc. Microwaves, Antennas and Prop., vol. 147, no. 6, pp. 463-468, Dec. 2000.

[2] J.S.McLean, H.Foltz, and R.Sutton, "Conditions for direction-independent distortion in UWB antennas," IEEE Trans. on Antennas and Propagation, vol. 54, no. 11, pp. 3178-3183, November 2006. 